\title{
Far-infrared $\mathrm{CO}$ and $\mathrm{H}_{2} \mathrm{O}$ emission in intermediate-mass protostars ${ }^{\star}$ (Research Note)
}

\author{
M. Matuszak ${ }^{1}$, A. Karska ${ }^{1}$, L. E. Kristensen ${ }^{2}$, G. J. Herczeg ${ }^{3}$, Ł. Tychoniec $^{1}$, T. A. van Kempen ${ }^{4}$, and A. Fuente ${ }^{5}$ \\ 1 Astronomical Observatory, Adam Mickiewicz University, Słoneczna 36, 60-268 Poznań, Poland \\ e-mail: agata.karska@gmail.com \\ 2 Harvard-Smithsonian Center for Astrophysics, 60 Garden Street, Cambridge, MA 02138, USA \\ 3 Kavli Institut for Astronomy and Astrophysics, Yi He Yuan Lu 5, HaiDian Qu, Peking University, 100871 Beijing, PR China \\ ${ }^{4}$ Leiden Observatory, Leiden University, PO Box 9513, 2300 RA Leiden, The Netherlands \\ 5 Observatorio Astronómico Nacional (OAN, IGN), Apdo 112, 28803 Alcalá de Henares, Spain
}

Received 4 March 2015 / Accepted 13 April 2015

\section{ABSTRACT}

\begin{abstract}
Context. Intermediate-mass young stellar objects (YSOs) provide a link to understanding how feedback from shocks and UV radiation scales from low- to high-mass star forming regions.

Aims. Our aim is to analyze excitation of $\mathrm{CO}$ and $\mathrm{H}_{2} \mathrm{O}$ in deeply embedded intermediate-mass YSOs and compare it with similar studies on low-mass and high-mass YSOs.

Methods. Herschel/PACS spectral maps are analyzed for six YSOs with bolometric luminosities of $L_{\mathrm{bol}} \sim 10^{2}-10^{3} L_{\odot}$. The maps cover spatial scales of $\sim 10^{4} \mathrm{AU}$ in several $\mathrm{CO}$ and $\mathrm{H}_{2} \mathrm{O}$ lines located in the $\sim 55-210 \mu \mathrm{m}$ range.

Results. Rotational diagrams of CO show two temperature components at $T_{\text {rot }} \sim 320 \mathrm{~K}$ and $T_{\text {rot }} \sim 700-800 \mathrm{~K}$, comparable to lowand high-mass protostars probed at similar spatial scales. The diagrams for $\mathrm{H}_{2} \mathrm{O}$ show a single component at $T_{\text {rot }} \sim 130 \mathrm{~K}$, as seen in low-mass protostars, and about $100 \mathrm{~K}$ lower than in high-mass protostars. Since the uncertainties in $T_{\text {rot }}$ are on the same order as the difference between the intermediate and high-mass protostars, we cannot conclude whether the change in rotational temperature occurs at a specific luminosity or whether the change is more gradual from low- to high-mass YSOs.

Conclusions. Molecular excitation in intermediate-mass protostars is comparable to the central $10^{3} \mathrm{AU}$ of low-mass protostars and consistent within the uncertainties with the high-mass protostars probed at $3 \times 10^{3} \mathrm{AU}$ scales, suggesting similar shock conditions in all those sources.
\end{abstract}

Key words. stars: protostars - ISM: jets and outflows - ISM: molecules

\section{Introduction}

Feedback processes associated with the collapse of protostellar envelopes at $10^{3}-10^{4} \mathrm{AU}$ scales limit the accretion onto the protostar and contribute to the overall low efficiency of transferring gas into stars on global scales (Offner et al. 2009; Krumholz et al. 2014). Calculating the physical conditions help to identify the most relevant phenomena and constrain their role in the star formation process (Evans 1999). Since gas in protostellar envelopes is heated to temperatures much higher than the dust temperatures, molecular transitions are the suitable tracers of physical conditions of hot $(T \gtrsim 100 \mathrm{~K})$ gas around protostars. In particular, the far-infrared (IR) lines of $\mathrm{CO}$ and $\mathrm{H}_{2} \mathrm{O}$ dominate the cooling of hot and dense gas (Goldsmith \& Langer 1978). The excitation of $\mathrm{CO}$ and $\mathrm{H}_{2} \mathrm{O}$ depends on the local physical conditions (temperature, density) and thus is crucial in order to determine which physical mechanisms are responsible for the gas heating and to study whether the energetics involved in the feedback scale from low- to intermediate- to high-mass young stellar objects (YSOs).

Recent observations of $\mathrm{CO}$ and $\mathrm{H}_{2} \mathrm{O}$ lines with the Photodetector Array Camera and Spectrometer (PACS, Poglitsch et al. 2010) on board Herschel found large columns

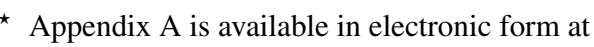
http://www. aanda.org
}

of dense $\left(\gtrsim 10^{4} \mathrm{~cm}^{-3}\right)$ and hot $(\gtrsim 300 \mathrm{~K})$ gas towards lowmass $\left(L_{\mathrm{bol}} \lesssim 10^{2} L_{\odot}\right)$ protostars (van Kempen et al. 2010; Herczeg et al. 2012; Manoj et al. 2013; Karska et al. 2013; Green et al. 2013; Lindberg et al. 2014) that originate largely from UV-irradiated shocks associated with jets and winds (Karska et al. 2014b). The $\mathrm{CO}$ and $\mathrm{H}_{2} \mathrm{O}$ line luminosities of the high-mass protostars (with $L_{\mathrm{bol}} \sim 10^{4}-10^{6} L_{\odot}$ ) follow the correlations with bolometric luminosities found in the low-mass protostars (Karska et al. 2014a) and show similar velocityresolved line profiles regardless of the mass of the protostar (Yildız et al. 2013; San José-García et al. 2013; van der Tak et al. 2013). In contrast, rotational temperatures of $\mathrm{H}_{2} \mathrm{O}$ are lower and the $\mathrm{H}_{2} \mathrm{O}$ fraction contributed to the total cooling in lines with respect to $\mathrm{CO}$ is higher for the low-mass protostars (Karska et al. 2014a; Goicoechea et al. 2015), suggesting that the physical mechanisms causing the excitation in low- and high-mass protostars are different.

Intermediate-mass YSOs (with $L_{\text {bol }} \sim 10^{2}-10^{3} L_{\odot}{ }^{1}$ ) provide a natural link between low- and high mass protostars, but their

\footnotetext{
1 We use bolometric luminosity as a proxy of the protostellar mass for practical and historical reasons, but we note that $L_{\text {bol }}$ changes significantly during the protostellar phase if the accretion is episodic (Young \& Evans 2005; Dunham et al. 2010). Moreover, some of our intermediate-mass sources may actually be a collection of unresolved low-mass protostars.
} 
Table 1. $\mathrm{CO}$ and $\mathrm{H}_{2} \mathrm{O}$ rotational excitation.

\begin{tabular}{lcccccccc}
\hline \hline \multirow{2}{*}{ Source } & \multirow{2}{*}{$L_{\text {bol }}$} & \multicolumn{2}{c}{ Warm CO } & \multicolumn{2}{c}{$\operatorname{Hot} \mathrm{CO}$} & \multicolumn{2}{c}{$\mathrm{H}_{2} \mathrm{O}$} \\
& $(\mathrm{pc})$ & $\left(L_{\odot}\right)$ & $T_{\text {rot }}(\mathrm{K})$ & $\log _{10} \mathcal{N}$ & $T_{\text {rot }}(\mathrm{K})$ & $\log _{10} \mathcal{N}$ & $T_{\text {rot }}(\mathrm{K})$ & $\log _{10} \mathcal{N}$ \\
\hline AFGL 490 & 1000 & 2000 & $300(20)$ & $51.6(0.1)$ & $\ldots$ & $\ldots$ & $90(100)$ & $48.3(1.2)$ \\
L 1641 S3 MMS1 & 465 & 70 & $325(30)$ & $49.9(0.1)$ & $\ldots$ & $\ldots$ & $150(100)$ & $46.8(0.1)$ \\
NGC 2071 & 422 & 520 & $310(30)$ & $51.2(0.1)$ & $700(50)$ & $50.1(0.1)$ & $135(100)$ & $47.9(0.1)$ \\
Vela 17 & 700 & 715 & $265(25)$ & $50.8(0.2)$ & $\ldots$ & $\ldots$ & $90(40)$ & $47.8(0.5)$ \\
Vela 19 & 700 & 776 & $335(50)$ & $50.4(0.2)$ & $\ldots$ & $\ldots$ & $130(80)$ & $47.2(0.4)$ \\
NGC 7129 FIRS 2 & 1250 & 430 & $370(45)$ & $50.7(0.2)$ & $710(70)$ & $49.9(0.2)$ & $130(50)$ & $47.8(0.1)$ \\
\hline
\end{tabular}

Notes. Distances and bolometric luminosities are taken from Wampfler et al. (2013) and references therein.

far-IR $\mathrm{CO}$ and $\mathrm{H}_{2} \mathrm{O}$ emission has only been studied for a single protostar position of NGC 7129 FIRS 2 (Fich et al. 2010) and the outflow position of NGC 2071 (Neufeld et al. 2014). CO emission alone was analyzed for two intermediate-mass protostars in Orion, HOPS 288 and 370 (Manoj et al. 2013). In this paper, we present the analysis of PACS spectra for the full sample of intermediate-mass protostars from the "Water in star forming regions with Herschel" (WISH) key program (van Dishoeck et al. 2011), including the maps of NGC 7129 and NGC 2071 centered on the YSO position. These results complement the work by Wampfler et al. (2013), which describes the $\mathrm{OH}$ excitation in our source sample and the sample of low- and highmass protostars for which $\mathrm{CO}$ and $\mathrm{H}_{2} \mathrm{O}$ emission is discussed in Karska et al. (2013, 2014a). The main question addressed is whether $\mathrm{CO}$ and $\mathrm{H}_{2} \mathrm{O}$ rotational temperatures differ from low- to high-mass protostars.

The paper is organized as follows. Section 2 briefly introduces the observations, Sect. 3 the excitation analysis using rotational diagrams, and Sect. 4 discusses the results.

\section{Observations}

Our sample includes six YSOs with bolometric luminosities from 70 to $2000 L_{\odot}$ and located at an average distance of $700 \mathrm{pc}$ (see Table 1). The sources were selected based on their small distances $(\lesssim 1 \mathrm{kpc})$ and location accessible for follow-up observations from the southern hemisphere (for more details see Sect. 4.4.2. in van Dishoeck et al. 2011). Spectroscopy for all sources was obtained with PACS as part of the WISH program. For observing details see Table A.1.

With PACS, we obtained single footprint spectral maps covering a field of view of $\sim 47^{\prime \prime} \times 47^{\prime \prime}$ and resolved into $5 \times 5$ array of spatial pixels (spaxels) of $\sim 9.4^{\prime \prime} \times 9.4^{\prime \prime}$ each. At the distance to the sources, the full array corresponds to spatial scales of $\sim 2-6 \times 10^{4} \mathrm{AU}$ in diameter, on the order of full maps of lowmass YSOs $\left(\sim 10^{4} \mathrm{AU}\right)$ in Karska et al. (2013) and the central spaxel spectra of more distant high-mass YSOs $\left(\sim 3 \times 10^{4} \mathrm{AU}\right)$ in Karska et al. (2014a).

The observations were taken in the line spectroscopy mode, which provide deep integrations of $0.5-2 \mu \mathrm{m}$ wide spectral regions within the $\sim 55-210 \mu \mathrm{m}$ PACS range. Two nod positions were used for chopping $3^{\prime}$ on each side of the source (for details of our observing strategy and basic reduction methods, see Karska et al. 2013). The full list of targeted $\mathrm{CO}$ and $\mathrm{H}_{2} \mathrm{O}$ lines and the calculated line fluxes are shown in Table A.2. The quality of the spectra is illustrated in Fig. A.1. We note that the simultaneous non-detections of the $\mathrm{H}_{2} \mathrm{O} 7_{16}-7_{07}$ line at $84.7 \mu \mathrm{m}$ and detections of the $\mathrm{H}_{2} \mathrm{O} 8_{18}-7_{07}$ line at $63.3 \mu \mathrm{m}$ in $\mathrm{L} 1641$, NGC 2071, and NGC 7129 FIRS 2 are related to the structure of the $\mathrm{H}_{2} \mathrm{O}$ energy levels and not the differences in the sensitivities of the instrument in the second- and third-order observations. The $8_{18}-7_{07}$ line is a backbone transition, with the Einstein A coefficient higher than the value for the $7_{16}-7_{07}$ line.

The data reduction was done using HIPE v.13 with Calibration Tree 65 and subsequent analysis with customized IDL programs (see, e.g., Karska et al. 2014b). The fluxes were calculated using the emission from the entire maps. Figure A.2 illustrates that both the line and continuum emission peaks approximately at the source position, with small shifts in continuum due to mispointing. The extent of the line emission usually follows the continuum pattern with the exception of Vela 17 where the line emission extends from NE to SW direction, while the continuum is centrally peaked. There is no contamination detected from the nearby sources or their outflows in the targeted regions.

\section{Rotational diagrams}

\subsection{Results}

Figure 1 shows rotational diagrams of $\mathrm{CO}$ calculated for all sources in the same way as in Karska et al. (2014a). The corresponding rotational temperatures, $T_{\text {rot }}$, and total numbers of emitting molecules, $\mathcal{N}$, are shown in Table 1.

All sources show a $300 \mathrm{~K}$, "warm" CO component (Manoj et al. 2013; Karska et al. 2013; Green et al. 2013), with a mean temperature of $\sim 320 \mathrm{~K}$ for the range of total numbers of emitting molecules, $\mathcal{N} \sim 10^{50}-10^{51}$. In addition, NGC 2071 and NGC 7129 show a "hot" CO component with temperatures of $\sim 700 \mathrm{~K}$ and $\mathcal{N} \sim 10^{50}$.

The rotational diagrams for $\mathrm{H}_{2} \mathrm{O}$, presented in Fig. 2, show a single component with a mean temperature of $\sim 120 \mathrm{~K}$. The scatter due to subthermal excitation and high opacities exceeds the uncertainties in the observed fluxes, similar to diagrams of low- and high-mass YSOs (for the discussion of both effects see Sect. 4.2.2 of Karska et al. 2014a). The corresponding numbers of emitting molecules are about 3 orders of magnitude lower than for $\mathrm{CO}, \mathcal{N} \sim 10^{47}-10^{48}$.

\subsection{Comparison to low- and high-mass sources}

Figure 3 shows a comparison between rotational temperatures obtained for $\mathrm{CO}$ and $\mathrm{H}_{2} \mathrm{O}$ for the intermediate-mass sources presented here and these quantities determined in the same way for low- and high-mass YSOs. The comparison is restricted only to the warm component seen in $\mathrm{CO}$ rotational diagrams owing to the low number of sources with detections of the hot CO component; those will be discussed in detail in Karska et al. (in prep.). 

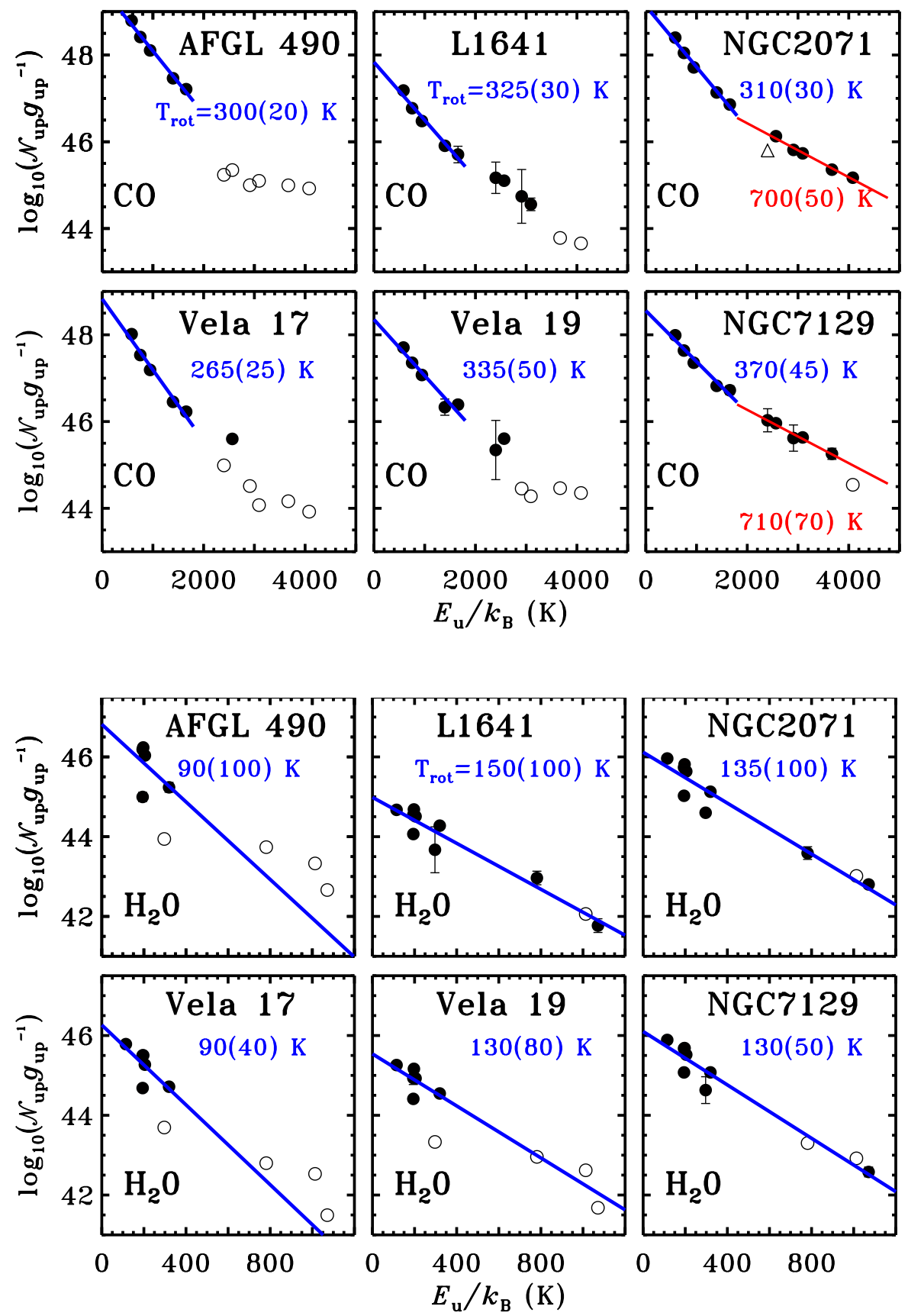

Fig. 1. Rotational diagrams of CO. The base-10 logarithm of the number of emitting molecules from the upper level, $\mathcal{N}_{\text {up }}$, divided by the degeneracy of the level, $g_{\text {up }}$, is shown as a function of energy of the upper level in kelvins, $E_{\text {up }}$. Detections are shown as filled circles, whereas three-sigma upper limits are shown as empty circles. The empty upper triangle corresponds to the line flux calculated using a smaller area on the map than the rest of the lines. Blue lines show linear fits to the data and the corresponding rotational temperatures. Errors associated with the least-square linear fit are shown in brackets.

Fig. 2. Similar to Fig. 1 but for $\mathrm{H}_{2} \mathrm{O}$.

A bolometric luminosity is used here as a proxy for the protostellar mass, but in fact some of our intermediate-mass sources may be a collection of unresolved low-mass protostars.

The median $T_{\text {rot }}$ of $\mathrm{CO}$ in low-mass protostars is $325 \mathrm{~K}$, using the results from the WISH (Karska et al. 2013), "Dust, Ice, and Gas in Time" (Green et al. 2013), and "Herschel Orion Protostar Survey" (Manoj et al. 2013) programs for a total of about 50 sources. A comparable value of $\sim 290 \mathrm{~K}$ was found for ten high-mass sources $\left(L_{\text {bol }} \sim 10^{4}-10^{6} L_{\odot}\right)$ in Karska et al. (2014a). The range of CO $T_{\text {rot }}$ of $265-370 \mathrm{~K}$ (see Table 1) determined here for intermediate-mass YSOs is thus fully consistent with previous results. The fact that the $300 \mathrm{~K} \mathrm{CO}$ component does not depend on the source bolometric luminosity over 6 orders of magnitude suggests that the origin is in a shock associated with the jet/winds impact on the envelope rather than in a photodissociation region where $T_{\text {rot }}$ should scale with the UV flux and luminosity (van Kempen et al. 2010; Visser et al. 2012; Manoj et al. 2013; Flower \& Pineau des Forêts 2013; Kristensen et al. 2013).

The median $\mathrm{H}_{2} \mathrm{O}$ rotational temperatures (see the bottom panel of Fig. 3) for low- and high-mass YSOs are $\sim 130 \mathrm{~K}$ and $\sim 230 \mathrm{~K}$, respectively (Karska et al. 2013, 2014a). The range of temperatures obtained for the intermediate-mass YSOs (90-150 K, Table 1) is thus comparable to the lowmass sources. However, the uncertainties in the rotational temperatures are high, on the order of $\sim 100 \mathrm{~K}$, and do not account for the optical depth effects, the density effects, and the possible complexity of the line profiles (absorption and emission components). It is therefore unclear if there is a true jump in rotational temperatures at $L_{\text {bol }} \sim 10^{4} L_{\odot}$, or a smooth trend 


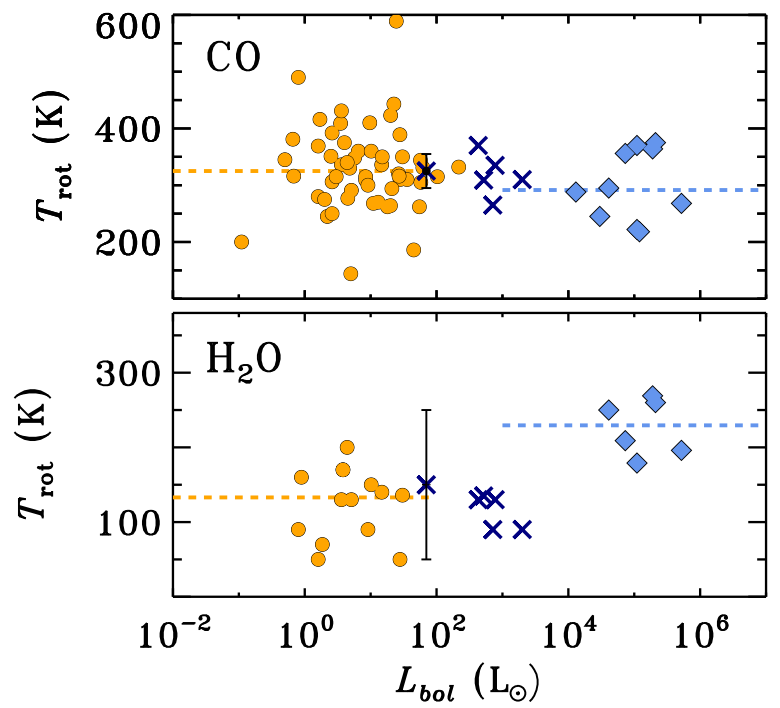

Fig. 3. Rotational temperatures of warm $\left(E_{\mathrm{up}}=14-24\right) \mathrm{CO}$ (top) and $\mathrm{H}_{2} \mathrm{O}$ (bottom) as function of bolometric luminosity. Orange circles show YSOs from Green et al. (2013), Karska et al. (2013), and Manoj et al. (2013). Dark blue crosses show intermediate-mass YSOs from the WISH program and light blue diamonds high-mass YSOs from Karska et al. (2014a). A few sources with luminosities $\sim 10^{2} L_{\odot}$ shown with orange crosses are from Manoj et al. (2013) and can also be regarded as intermediate-mass YSOs. Uncertainties of the $\mathrm{CO}$ and $\mathrm{H}_{2} \mathrm{O}$ rotational temperatures are shown for L1641 S3 MMS1, which is representative of the sample.

toward higher values of $T_{\text {rot }}$ In either case, these higher excitation temperatures could be due to higher densities in the more massive envelopes.

\section{Summary}

We analyze the excitation of far-IR $\mathrm{CO}$ and $\mathrm{H}_{2} \mathrm{O}$ lines in six intermediate-mass YSOs observed in the WISH survey and compare the results to low- and high-mass protostars. Rotational temperatures of $\mathrm{CO}$ and $\mathrm{H}_{2} \mathrm{O}$ are found to be $\sim 320 \mathrm{~K}$ and $\sim 120 \mathrm{~K}$, respectively, and are consistent with low-mass and high-mass YSOs within the uncertainties. The large uncertainties in the $\mathrm{H}_{2} \mathrm{O}$ rotational temperatures, on the order of $100 \mathrm{~K}$, and the order of magnitude gap in the bolometric luminosity between intermediate- and high-mass protostars does not allow us to conclude whether the changes are a smooth function of luminosity. Still, the similarities in rotational temperatures seen for sources with luminosities spanning 6 orders of magnitude and probed at different spatial scales strongly suggest the same excitation mechanism, the UV-irradiated shocks associated with jets and winds for all sources across the luminosity range (Kristensen et al. 2013; Karska et al. 2014b; Mottram et al. 2014).

Acknowledgements. The authors would like to thank the referee for the valuable comments which helped to improve the manuscript. Herschel is an ESA space observatory with science instruments provided by European-led Principal Investigator consortia and with important participation from NASA. A.K. acknowledges support from the Polish National Science Center grant 2013/11/N/ST9/00400. Research conducted within the scope of the HECOLS International Associated Laboratory, supported in part by the Polish NCN grant Dec-2013/08/M/ST9/00664.

\section{References}

Dunham, M. M., Evans, II, N. J., Terebey, S., Dullemond, C. P., \& Young, C. H. 2010, ApJ, 710, 470

Evans, II, N. J. 1999, ARA\&A, 37, 311

Fich, M., Johnstone, D., van Kempen, T. A., et al. 2010, A\&A, 518, L86 Flower, D. R., \& Pineau des Forêts, G. 2013, MNRAS, 436, 2143

Goicoechea, J. R., Chavarría, L., Cernicharo, J., et al. 2015, ApJ, 799, 102

Goldsmith, P. F., \& Langer, W. D. 1978, ApJ, 222, 881

Green, J. D., Evans, II, N. J., Jørgensen, J. K., et al. 2013, ApJ, 770, 123

Herczeg, G. J., Karska, A., Bruderer, S., et al. 2012, A\&A, 540, A84

Karska, A., Herczeg, G. J., van Dishoeck, E. F., et al. 2013, A\&A, 552, A141

Karska, A., Herpin, F., Bruderer, S., et al. 2014a, A\&A, 562, A45

Karska, A., Kristensen, L. E., van Dishoeck, E. F., et al. 2014b, A\&A, 572, A9

Kristensen, L. E., van Dishoeck, E. F., Benz, A. O., et al. 2013, A\&A, 557, A23

Krumholz, M. R., Bate, M. R., Arce, H. G., et al. 2014, Protostars and Planets

VI, University of Arizona Press, eds. H. Beuther, R. Klessen, C. Dullemond, \& Th. Henning, 243

Lindberg, J. E., Jørgensen, J. K., Green, J. D., et al. 2014, A\&A, 565, A29

Manoj, P., Watson, D. M., Neufeld, D. A., et al. 2013, ApJ, 763, 83

Mottram, J. C., Kristensen, L. E., van Dishoeck, E. F., et al. 2014, A\&A, 572, A21

Müller, H. S. P., Thorwirth, S., Roth, D. A., \& Winnewisser, G. 2001, A\&A, 370, L49

Müller, H. S. P., Schlöder, F., Stutzki, J., \& Winnewisser, G. 2005, J. Mol. Struct., 742,215

Neufeld, D. A., Gusdorf, A., Güsten, R., et al. 2014, ApJ, 781, 102

Offner, S. S. R., Klein, R. I., McKee, C. F., \& Krumholz, M. R. 2009, ApJ, 703, 131

Pickett, H. M., Poynter, R. L., Cohen, E. A., et al. 1998, J. Quant. Spectr. Rad. Transf., 60, 883

Poglitsch, A., Waelkens, C., Geis, N., et al. 2010, A\&A, 518, L2

San José-García, I., Mottram, J. C., Kristensen, L. E., et al. 2013, A\&A, 553, A125

van der Tak, F. F. S., Chavarría, L., Herpin, F., et al. 2013, A\&A, 554, A83

van Dishoeck, E. F., Kristensen, L. E., Benz, A. O., et al. 2011, PASP, 123, 138

van Kempen, T. A., Kristensen, L. E., Herczeg, G. J., et al. 2010, A\&A, 518, L121

Visser, R., Kristensen, L. E., Bruderer, S., et al. 2012, A\&A, 537, A55

Wampfler, S. F., Bruderer, S., Karska, A., et al. 2013, A\&A, 552, A56

Y1ldı, U. A., Kristensen, L. E., van Dishoeck, E. F., et al. 2013, A\&A, 556, A89

Young, C. H., \& Evans, II, N. J. 2005, ApJ, 627, 293 


\section{Appendix A: Supplementary material}

Table A.1 shows the observing log of PACS observations including observations identification numbers (OBSID), observation day (OD), date of observation, total integration time, and pointed coordinates (RA, Dec). Table A.2 shows molecular data and observed lines fluxes and upper limits for all sources that are analyzed in the paper. Figure A.1 shows selected spectral regions to illustrate the quality of the data. Figure A.2 illustrates the patterns of continuum emission at $145 \mu \mathrm{m}$ and the CO $18-17$ line emission at $144 \mu \mathrm{m}$ toward all the sources.

Table A.1. Log of PACS observations.

\begin{tabular}{lcccccc}
\hline \hline Source & OBSID & OD & Date & $\begin{array}{c}\text { Total time } \\
(\mathrm{s})\end{array}$ & $\begin{array}{c}\text { RA } \\
(\mathrm{h} \mathrm{m} \mathrm{s})\end{array}$ & $\begin{array}{c}\text { Dec } \\
\left({ }^{\circ} \prime^{\prime \prime}\right)\end{array}$ \\
\hline AFGL 490 & 1342202582 & 454 & 2010-Aug.-11 & 3882 & 32738.40 & +584708.0 \\
& 1342191353 & 290 & 2010-Feb.-28 & 2029 & 32738.40 & +584708.0 \\
L1641 S3 MMS1 & 1342226194 & 823 & 2011-Aug.-14 & 3882 & 53955.90 & -73028.0 \\
& 1342226195 & 823 & 2011-Aug.-14 & 1987 & 53955.90 & -73028.0 \\
NGC 2071 & 1342218760 & 703 & 2011-Apr.-17 & 3882 & 54704.40 & +02149.0 \\
& 1342218761 & 703 & 2011-Apr.-17 & 1987 & 54704.40 & +02149.0 \\
Vela IRS 17 & 1342209407 & 551 & 2010-Nov.-16 & 3882 & 84634.70 & -435430.5 \\
& 1342211844 & 594 & 2010-Dec.-28 & 1987 & 84634.70 & -435430.5 \\
Vela IRS 19 & 1342210189 & 552 & 2010-Nov.-16 & 3882 & 84848.50 & -433229.0 \\
& 1342210190 & 552 & 2010-Nov.-16 & 1987 & 84848.50 & -433229.0 \\
NGC 7129 FIRS2 & 1342186321 & 165 & 2009-Oct.-26 & 3895 & 214301.70 & +660323.6 \\
& 1342186322 & 165 & 2009-Oct.-26 & 2000 & 214301.70 & +660323.6 \\
\hline
\end{tabular}

Table A.2. Molecular data ${ }^{a}$ and observed line fluxes.

\begin{tabular}{|c|c|c|c|c|c|c|c|c|c|c|c|}
\hline Species & Transition & $\begin{array}{l}\text { Wave. } \\
(\mu \mathrm{m})\end{array}$ & $\begin{array}{c}\text { Rest Freq. } \\
\quad(\mathrm{GHz})\end{array}$ & $\begin{array}{c}E_{\mathrm{u}} / k_{\mathrm{B}} \\
(\mathrm{K})\end{array}$ & $\begin{array}{c}A_{\mathrm{ul}} \\
\left(\mathrm{s}^{-1}\right)\end{array}$ & AFGL 490 & L 1641 & $\begin{array}{l}\text { NGC } 2071 \\
\quad \text { Fluxes }(1\end{array}$ & $\begin{array}{c}\text { Vela } 17 \\
\left.0^{-20} \mathrm{~W} \mathrm{~cm}^{-2}\right)\end{array}$ & Vela 19 & 7129 FIRS 2 \\
\hline$\overline{\mathrm{CO}}$ & $14-13$ & 185.999 & 1611.8 & 580.5 & $2.7(-4)$ & $11.01 \pm 0.11$ & $5.00 \pm 0.10$ & $100.13 \pm 0.69$ & $14.92 \pm 0.15$ & $7.38 \pm 0.10$ & $4.41 \pm 0.07$ \\
\hline $\mathrm{H}_{2} \mathrm{O}$ & $2_{21}-2_{12}$ & 180.488 & 1661.0 & 194.1 & $3.1(-2)$ & $1.62 \pm 0.07$ & $0.69 \pm 0.06$ & $12.95 \pm 0.09$ & $2.74 \pm 0.06$ & $0.74 \pm 0.12$ & 0.05 \\
\hline $\mathrm{H}_{2} \mathrm{O}$ & $22_{12}-1_{01}$ & 179.527 & 1669.9 & 114.4 & $5.6(-2)$ & absorption & $1.69 \pm 0.06$ & $39.81 \pm 0.40$ & $9.65 \pm 0.08$ & $2.88 \pm 0.06$ & $3.85 \pm 0.08$ \\
\hline $\mathrm{H}_{2} \mathrm{O}$ & $3_{03}-2_{12}$ & 174.626 & 1716.8 & 196.8 & $5.1(-2)$ & $4.36 \pm 0.08$ & $2.25 \pm 0.06$ & $36.97 \pm 0.30$ & $6.58 \pm 0.07$ & $3.01 \pm 0.11$ & 0.07 \\
\hline $\mathrm{CO}$ & $16-15$ & 162.812 & 1841.3 & 751.7 & $4.1(-4)$ & $8.84 \pm 0.11$ & $3.76 \pm$ & $85.61 \pm 0.45$ & $9.48 \pm 0.18$ & $6.32=$ & 0.07 \\
\hline $\mathrm{CO}$ & $18-17$ & 144.784 & 2070.6 & 945.0 & $5.7(-4)$ & 7.74 & $3.36=$ & $70.00 \pm$ & 7.62 & 5.84 & .13 \\
\hline $\mathrm{H}_{2} \mathrm{O}$ & $3_{13}-2_{02}$ & 138.528 & 2164.1 & 204.7 & $1.3(-1)$ & $2.86 \pm 0.08$ & $1.57 \pm 0.07$ & $25.60 \pm 0.14$ & $3.99 \pm 0.06$ & 1.8 & 0.06 \\
\hline $\mathrm{H}_{2} \mathrm{O}$ & $4_{04}-3_{13}$ & 125.354 & 2391.6 & 319.5 & $1.7(-1)$ & $0.89 \pm 0.11$ & $1.80 \pm 0.05$ & $15.52 \pm 0.20$ & $2.19 \pm 0.10$ & $1.48 \pm 0.11$ & 0.08 \\
\hline $\mathrm{CO}$ & $22-21$ & 118.581 & 2528.2 & 1397.4 & $1.0(-3)$ & $4.61 \pm 0.15$ & $2.37 \pm 0.11$ & $49.00 \pm 0.26$ & $3.68 \pm 0.15$ & $2.78 \pm 0.52$ & $2.70 \pm 0.11$ \\
\hline $\mathrm{CO}$ & $24-23$ & 108.763 & 2756.4 & 1656.5 & $1.3(-3)$ & $3.91 \pm 0.34$ & $2.25 \pm 0.43$ & $38.80 \pm 0.96$ & $3.30 \pm 0.29$ & 4.80 & 0.33 \\
\hline $\mathrm{H}_{2} \mathrm{O}$ & $2_{21}-1_{10}$ & 108.073 & 2774.0 & 194.1 & $2.6(-1)$ & $1.47 \pm 0.17$ & $3.19 \pm 0.14$ & $35.30 \pm 0.30$ & $5.83 \pm 0.16$ & 0.16 & 0.12 \\
\hline $\mathrm{CO}$ & $29-28$ & 90.163 & 3325.0 & 2399.8 & $2.1(-3)$ & $<0.1$ & $1.58 \pm 0.57$ & $>8.17$ & $<0.46$ & $1.04 \pm 0.71$ & 0.42 \\
\hline $\mathrm{H}_{2} \mathrm{O}$ & $3_{22}-2_{11}$ & 89.988 & 31.5 & 296.8 & $3.5(-1)$ & $<0.1$ & $0.99 \pm 0.57$ & $10.21 \pm 0.30$ & $<0.46$ & $<0.20$ & 0.42 \\
\hline $\mathrm{CO}$ & $30-29$ & 87.190 & 38.4 & 2564.9 & $2.3(-3)$ & $<0.15$ & $1.58 \pm 0.09$ & $20.37 \pm 0.13$ & $2.19 \pm 0.19$ & $2.22 \pm 0.16$ & 0.07 \\
\hline $\mathrm{H}_{2} \mathrm{O}$ & $7_{16}-7_{07}$ & 84.767 & 36.7 & 1013.2 & $2.1(-1)$ & 1 & $<0.10$ & $<1.09$ & $<0$ & $<0.16$ & 0.10 \\
\hline $\mathrm{CO}$ & $32-31$ & 81.806 & 3664.7 & 2911.2 & $2.7(-3)$ & $<0.1$ & $0.92 \pm 0.57$ & $13.16 \pm 0.40$ & $<0.24$ & 21 & $0.96 \pm 0.29$ \\
\hline $\mathrm{CO}$ & $33-32$ & 79.360 & 3777.6 & 3092.5 & $3.0(-3)$ & $<0.13$ & $0.48 \pm 0.07$ & $12.56 \pm 0.24$ & $<0.10$ & & 0.09 \\
\hline $\mathrm{H}_{2} \mathrm{O}$ & $6_{15}-5_{24}$ & 78.928 & 3798.3 & 781.1 & $4.6(-1)$ & 17 & $0.53 \pm 0.09$ & $2.71 \pm$ & $<0.16$ & 23 & \\
\hline $\mathrm{CO}$ & $36-35$ & 72.843 & 4115.6 & 3668.8 & $3.6(-3)$ & & $<0.04$ & $7.74 \pm$ & $<0.18$ & 36 & $0.70 \pm 0.09$ \\
\hline $\mathrm{CO}$ & $38-37$ & 69.074 & & 4080.0 & $4.1(-3)$ & & & & & & \\
\hline $\mathrm{H}_{2} \mathrm{O}$ & $8_{18}-7_{07}$ & 63.324 & 4734.3 & 1070.7 & $1.8(0)$ & $<0.27$ & $0.64 \pm 0.11$ & $8.36 \pm 0.37$ & $<0.15$ & $<0.23$ & $0.57 \pm 0.07$ \\
\hline
\end{tabular}

Notes. Compiled using the CDMS (Müller et al. 2001, 2005) and JPL (Pickett et al. 1998) databases. Values of the Einstein $A$ coefficient are written in a form $A(B) \equiv A \times 10^{B}$. 


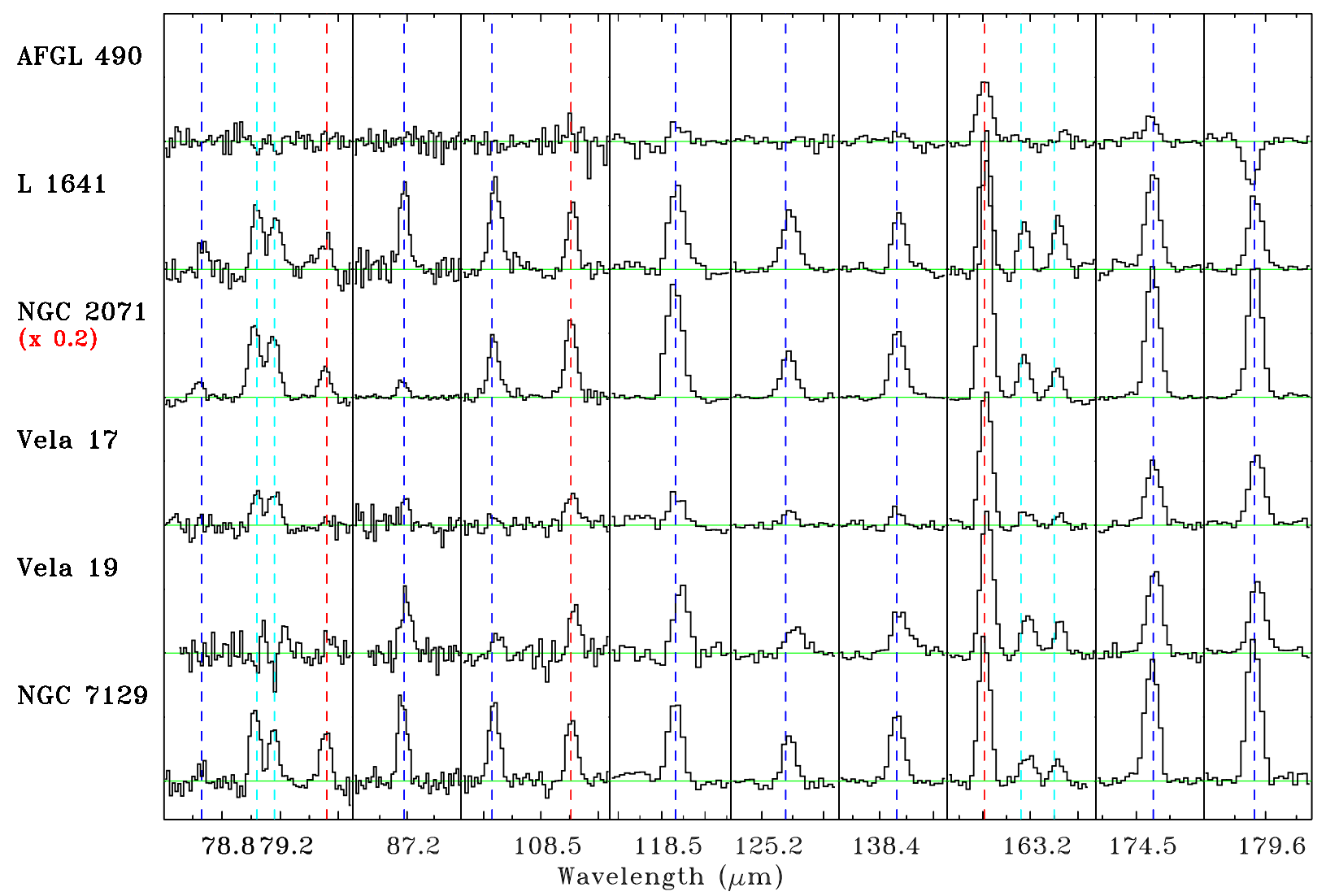

Fig. A.1. Spectral scans covering selected $\mathrm{H}_{2} \mathrm{O}, \mathrm{CO}$, and $\mathrm{OH}$ lines in the intermediate-mass protostars from the WISH program. The rest wavelength of each line is indicated by dashed lines: blue for $\mathrm{H}_{2} \mathrm{O}$, red for $\mathrm{CO}$, and light blue for $\mathrm{OH}$. 
M. Matuszak et al.: Intermediate-mass YSOs with PACS $(R N)$
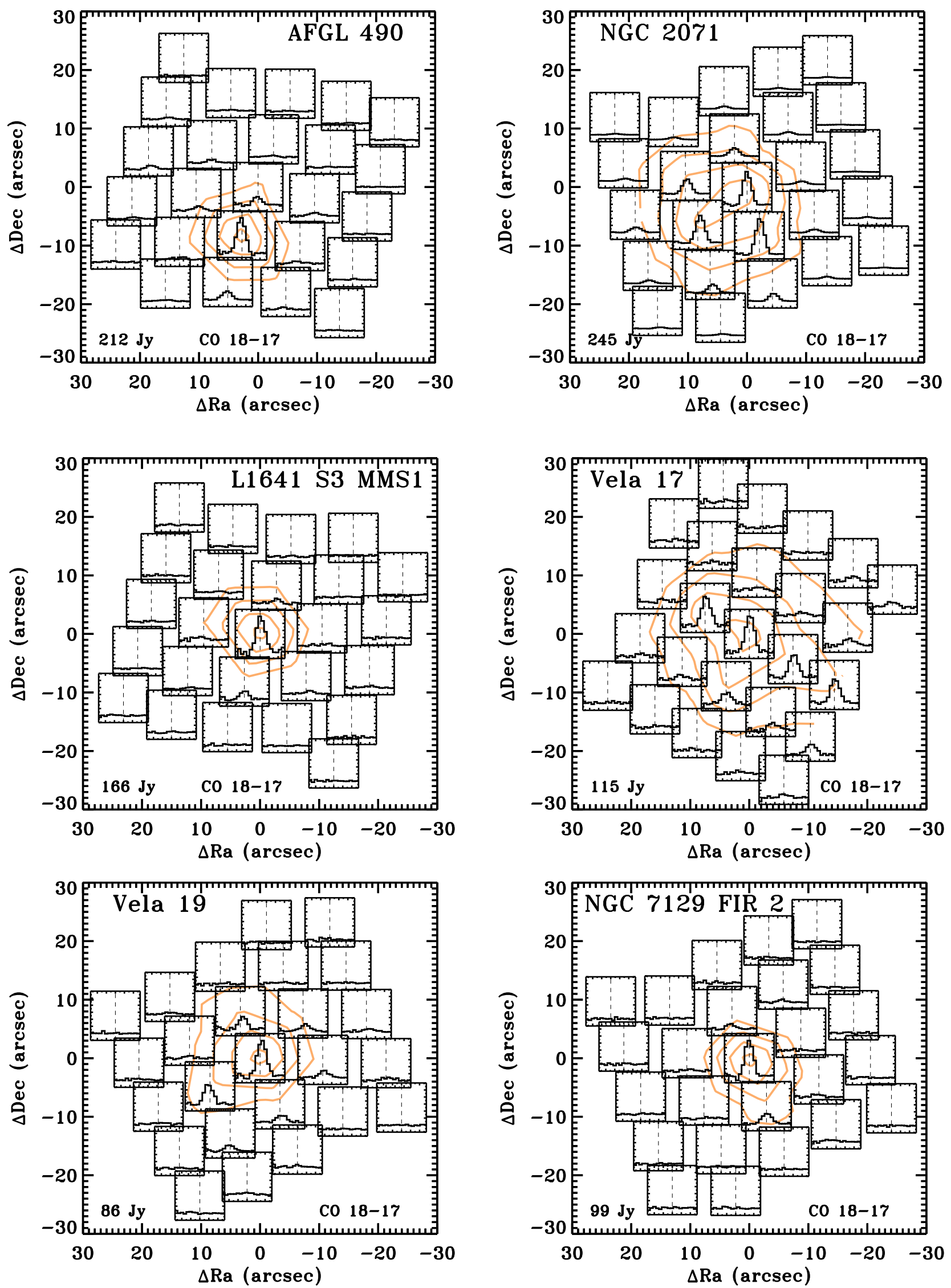

Fig. A.2. PACS spectral maps in the CO 18-17 line at $144 \mu \mathrm{m}$ and the continuum emission at $145 \mu \mathrm{m}$ in orange contours corresponding to $30 \%$, $50 \%, 70 \%$, and $90 \%$ of the peak value written in the bottom left corner of each map. Wavelengths in microns are translated to the velocity scale on the $X$-axis using laboratory wavelengths (see Table A.2) of the species and cover the range from -600 to $600 \mathrm{~km} \mathrm{~s}^{-1}$. The $Y$-axis shows fluxes in Jy normalized to the spaxel with the brightest line on the map in a range from -0.2 to 1.2. 